\title{
Predictive Control for Tight Group Formation of Multi-Agent Systems *
}

\author{
Ionela Prodan *** Sorin Olaru* Cristina Stoica * \\ Silviu-Iulian Niculescu ${ }^{* *}$ \\ * SUPELEC Systems Sciences (E3S) - Automatic Control Department, \\ France, (\{ionela.prodan,sorin.olaru,cristina.stoica\}@supelec.fr). \\ ** SUPELEC - CNRS - Laboratory of Signal and Systems, France, \\ (\{ionela.prodan,silviu.niculescu\}@lss.supelec.fr).
}

\begin{abstract}
This paper addresses the real-time control problem of a group of agents in the presence of non-convex collision avoidance constraints. The goal is to guarantee the convergence towards a tight formation. Convex (polyhedral) regions will be used to define safety regions around each agent. Then, using the dynamics and constraints, an optimization-based control design can be adopted upon an appropriate receding horizon principle. A single optimal control problem is solved based on a prediction of the future evolution of the system and the resulting control law is implemented in a centralized way. At the supervision level, it is shown that the decision about which agents should take on what role in the desired tight formation is equivalent with a classical pairing (or task assignment) problem. An important contribution is the re-evaluation of the pairing at each iteration. Copyright (C)IFAC 2011
\end{abstract}

Keywords: Multi-Agent Systems, constrained MPC, non-convex constraints, ultimate bounds

\section{INTRODUCTION}

In many applications, maintaining a formation is fundamental, for example in multiple satellite where the formation is used for astronomical observations and science missions [Mesbahi and Hadaegh, 2001], or in multiple aircraft where the formation is used to explore aerodynamic effects [Blake and Multhopp, 1998]. Questions about the characterization and the convergence towards a formation represents classical concerns for multi-agent systems. The problems become even more challenging if one needs to ensure that all the agents avoid collisions inside the group. Consequently, these problems are relevant in many applications involving the control of cooperative systems [Dunbar and Murray, 2002].

The main goal of this paper is to control a set of agents having independent dynamics while achieving a global objective, such as a tight formation with desired specifications and collision free behavior.

The collision avoidance between the agents is known to be a difficult problem, since certain constraints are non-convex. A popular framework for the treatment of such decision problems is represented by Mixed-IntegerProgramming (MIP) [Osiadacz et al., 1990]. The main motivation for using mixed-integer techniques is their ability to include non-convex constraints and discrete decisions in the optimization problem. There is a large literature dedicated to the design of multi-agent cooperative control strategies. For example, [Osiadacz et al., 1990],[Richards and How, 2002] detailed the use of MIP for "off-line" trajectory design with collision avoidance constraints. In

* The research of Ionela Prodan is financially supported by the EADS Foundation (091-AO09-1006). a different framework, [Bemporad and Morari, 1999] proposed the combination of MIP and Model Predictive Control (MPC) to stabilize general hybrid systems around equilibrium points. Finally, hierarchical methods are used for multi-vehicle target assignment and intercept problems in [Beard et al., 2002], [Earl and D'Andrea, 2007]. It is well known that mixed-integer techniques are NP-hard [Garey and Johnson, 1979], i.e. the computational complexity increases exponentially with the number of binary variables used in the problem formulation. Therefore, an important contribution of this work is that we formulate the problem using fewer binary variables through a more compact codification of the inequalities describing the feasible region Stoican et al. [2011].

The contributions of the present paper are on the one hand reducing the computational cost and on the other hand efficiently handling an increased number of constraints. To the best of the authors' knowledge there does not exist any similar methods and approaches in the open literature.

In this paper, the ideal formation is described starting from the definition of ultimate bounds for the dynamics in presence of additive disturbances, e.g. [Kofman et al., 2007]. This choice is adopted in order to guarantee a degree of robustness despite the fact that the real-time control is performed using nominal prediction models. Once the positions in the formation are established, the safety regions are defined around the nodes of the formation.

The formation control problem is decomposed in two separate problems:

- The "off-line" definition of the ideal configuration. A minimal configuration is determined with respect to a given cost function under the constraints imposed by the safety regions. 
- In real-time, a receding horizon optimization combined with task assignment relative to the minimal configuration will be employed.

The real-time control is designed based on the following two-stage procedure:

(1) Determine "who-goes-where" in the formation. This is equivalent with solving a standard assignment problem, which is a special case of the so-called Hitchcock Transportation Problem (TP) [Hallefjord, 1993].

(2) Solve a mixed-integer optimization problem according to the target geometry of the formation and the associated safety regions.

Finally, this two separate problems are embedded within a model predictive control problem, leading to an optimization problem for driving the group of agents to a specified formation with associated target locations.

The rest of the paper is organized as follows. Section 2 describes the individual agents model and the ultimate bound concept. Considering the nominal dynamics of the agents, in Section 3, we state the mixed-integer optimization problem to determine a desired target formation for the group of agents and then, use the result for formulating the assignment problem. Further on, in Section 5 we formulate the optimal control problem embedded within MPC, which explicitly involves the agents dynamics. Finally, several concluding remarks are drawn in Section 6 .

The following notations will be used throughout the paper: Denote by $\mathbb{N}_{[a, b]}$ the set of consecutive natural numbers between $a$ and $b$. Minkowski's addition of two sets $\mathcal{X}$ and $\mathcal{Y}$ is defined as $\mathcal{X} \oplus \mathcal{Y}=\{A+B: A \in \mathcal{X}, B \in \mathcal{Y}\}$. Denote $\mathbb{R}^{n}$ the space of real numbers and the projection from $\mathbb{R}^{n+p}$ to the first $p$ coordinates $\mathbb{R}^{p}$ as $\operatorname{Proj}_{p}($.$) .$ The closure of a set $S, \operatorname{cl}(S)$ is the intersection of all closed sets containing $S$. The collection of all possible $n_{c}$ combinations of binary variables will be noted $\{0,1\}^{n_{c}}=\left\{\left(b_{1}, \ldots, b_{n_{c}}\right): b_{i} \in\{0,1\}, i=1, \ldots, n_{c}\right\}$. Denote as $\mathbb{B}_{p}^{n}=\left\{x \in \mathbb{R}^{n}:\|x\|_{p} \leq 1\right\}$ the unit ball of norm $p$ where $\|x\|_{p}$ is the $p$-norm of vector $x$.

\section{PRELIMINARIES AND PREREQUISITES}

In the sequel, several basic concepts are revisited in order to provide a systematic procedure for describing a minimal configuration for the group of agents. A set of $N_{a}$ linear systems (vehicles, pedestrians or agents in a general form) will be used to model the behavior of individual agents. The $i^{t h}$ system is described by the following discrete-time invariant dynamics affected by additive disturbances:

$$
x_{k+1_{d}}^{i}=A_{i} x_{k_{d}}^{i}+B_{i} u_{k_{d}}^{i}+w_{k}^{i}, \quad i \in \mathbb{N}_{\left[1, N_{a}\right]},
$$

where $x_{k_{d}}^{i} \in \mathbb{R}^{n}$ are the state variables, $u_{k_{d}}^{i} \in \mathbb{R}^{m}$ is the control input vector and $w_{k}^{i} \in \mathbb{R}^{n}$ represents a bounded disturbance for the agent $i$. Henceforth we assume the following:

(1) The pairs $\left(A_{i}, B_{i}\right)$ are stabilizable, with $A_{i} \in \mathbb{R}^{n \times n}$, $B_{i} \in \mathbb{R}^{n \times m}$.

(2) The disturbance $w^{i}$ is bounded, i.e. $w \in \mathcal{W}$, where $\mathcal{W}$ is a convex and compact set containing the origin.
The nominal systems corresponding to (1) for the $i^{\text {th }}$ system is defined by:

$$
x_{k+1}^{i}=A_{i} x_{k}^{i}+B_{i} u_{k}^{i}, \quad i \in \mathbb{N}_{\left[1, N_{a}\right]} .
$$

Remark 1. The system (1) will be used in oder to describe the characteristics of the minimal group formation at the "off-line" stage. The control defines the target positions and the shape of the safety region around them. The nominal system (2) is used "on-line" for the optimizationbased real-time control. This will provide a computational efficient nominal trajectory. The state of the real system is then expected to reside in a tube around this nominal trajectory [Raković et al., 2005], [Mayne et al., 2005].

\subsection{Prerequisites}

The following definitions in set invariance analysis are necessary throughout the paper.

Definition 1. ([Blanchini and Miani, 2007]) A set $S_{i}$ is called robustly positive invariant (RPI) for the discretetime system (1), if $A_{i} x_{k_{d}}^{i}+B_{i} u_{k_{d}}^{i}+w_{k}^{i} \in S_{i}$ for all $x_{k_{d}}^{i} \in S_{i}$, $w_{k}^{i} \in \mathcal{W}$, with $i \in \mathbb{N}_{\left[1, N_{a}\right]}$, i.e. if

$$
\left(A_{i}+B_{i} K_{i}\right) S_{i} \oplus \mathcal{W} \subseteq S_{i} .
$$

Definition 2. ([Blanchini and Miani, 2007]) A set $\Omega_{\infty}$ is called minimal robustly invariant (mRPI) for (1) if it is a RPI set in $\mathbb{R}^{n}$ contained in every RPI set for (1).

Remark 2. The existence of a stabilizable pair $\left(A_{i}, B_{i}\right)$ implies the existence of an optimal control law for each agent $i, K_{i} \in \mathbb{R}^{n \times m}$ such that the matrices $A_{i}+B_{i} K_{i}$ are stable, where the controller $K_{i}, i \in \mathbb{N}_{\left[1, N_{a}\right]}$ is constructed either by a LQ design using the solution of the discrete algebraic Riccati equation or alternatively by pole placement technique. In both cases the existence of a quadratic Lyapunov function is supposed to account for the infinitetime cost function.

Taking into account the above remarks, the control law for the $i^{\text {th }}$ system defined along the lines in [Mayne et al., 2005] is used:

$$
u_{k_{d}}^{i}=u_{k}^{i}+K_{i}\left(x_{k_{d}}^{i}-x_{k}^{i}\right),
$$

such that the tracking error of the $i^{\text {th }}$ nominal system (2) is described as $z_{k}^{i}=x_{k_{d}}^{i}-x_{k}^{i}$, with the dynamics:

$$
z_{k+1}^{i}=\left(A_{i}+B_{i} K_{i}\right) z_{k}^{i}+w_{k}^{i} .
$$

Using this tracking error information, it follows that an RPI set $S_{z_{i}}$ can be determined and the following expression holds:

$$
x_{k_{d}}^{i}-x_{k}^{i} \in S_{z_{i}}
$$

for all $z_{0}^{i} \in S_{z_{i}}$ and $k \geq 0$.

With these basic remarks, note that it is sufficient to adjust the trajectory based on the nominal system (2), which does not depend on disturbance. This always guarantees that the real system (1) resides in a tube

$$
x_{k_{d}}^{i} \in x_{k}^{i} \oplus S_{z_{i}}
$$

along the reference path $x_{k}^{i}$ for all $u_{k}^{i}$.

There are various algorithms able to offer arbitrary close outer approximation of the mRPI set associated to (5) (as for example, the approaches proposed by [Raković et al., 2005], [Olaru et al., 2008]). It is worth mentioning that, these algorithms ignore the exponentially increase 
in the complexity of the representation. To overcome this inconvenient, an ultimate bound construction is further used, as proposed in [Kofman et al., 2007] due to its low computational demands. The following theorem from [Kofman et al., 2007] is recalled here as an instrumental result for the linear class of systems (1).

Theorem 1. [Kofman et al., 2007] Consider a system $x_{k+1}=A x_{k}+w$ and let $A=V J V^{-1}$ be the Jordan decomposition of $A$. Consider also a nonnegative vector $\bar{w}$ such that $|w| \leq \bar{w}, \forall w \in \mathcal{W} \subset \mathbb{R}^{n}$. Then the set

$$
\left.\Omega_{U B}=\left\{x \in \mathbb{R}^{n}:\left|V^{-1} x\right| \leq(I-|J|)^{-1}\right)\left|V^{-1}\right| \bar{w}\right\}
$$

is robustly positive invariant with respect to the dynamics $x_{k+1}=A x_{k}+w$.

For the collision avoidance problem, the nominal dynamics (2) will be used, but it is required that the real agents do not intersect. Since their true position is unknown (due to the disturbance affecting them), we can however be certain that an agent $i$ will always reside in a region

$$
\mathcal{S}_{i}\left(x_{k}^{i}\right) \triangleq x_{k}^{i} \oplus \Omega_{U B}^{i},
$$

where $\Omega_{U B}^{i}$ is the RPI set under the dynamics (5), as in Theorem 1, with $i \in \mathbb{N}_{\left[1, N_{a}\right]}$.

Remark 3. For the ease of the computation, the agents are considered as unidimensional points in the position space. Even if they are characterized by a nonempty region $R_{i} \subset \mathbb{R}^{n}$, one can define the set in (7) as

$$
\tilde{\mathcal{S}}_{i}\left(x_{k}^{i}\right) \triangleq \mathcal{S}_{i}\left(x_{k}^{i}\right) \oplus R_{i}
$$

where $R_{i}$ denotes the region describing the $i^{\text {th }}$ agent.

\subsection{Exemplification for construction of the RPI sets}

Consider a set of three heterogeneous agents in two spatial dimensions with the dynamics described by:

$$
A_{i}=\left[\begin{array}{cccc}
0 & 0 & 1 & 0 \\
0 & 0 & 0 & 1 \\
0 & 0 & -\frac{\mu_{i}}{m_{i}} & 0 \\
0 & 0 & 0 & -\frac{\mu_{i}}{m_{i}}
\end{array}\right], B_{i}=\left[\begin{array}{cc}
0 & 0 \\
0 & 0 \\
\frac{1}{m_{i}} & 0 \\
0 & \frac{1}{m_{i}}
\end{array}\right]
$$

affected by the additive disturbances:

$$
\left|w_{i}\right|<\left[\begin{array}{llll}
0.5 & 0.3 & 0.5 & 0.2
\end{array}\right]^{T} \text {. }
$$

where $\left[\begin{array}{llll}x^{i} & y^{i} & v_{x}^{i} & v_{y}^{i}\end{array}\right]^{T},\left[\begin{array}{ll}u_{x}^{i} & u_{y}^{i}\end{array}\right]^{T}$ are the state and the input of each system. The components of the state are: the position $\left(x^{i}, y^{i}\right)$ and the velocity $\left(v_{x}^{i}, v_{y}^{i}\right)$ of agent $i, i \in \mathbb{N}_{[1,3]}$. The parameters $m_{i}, \mu_{i}$ are the mass of the agent $i$ and the damping factor, respectively: $m_{1}=45 \mathrm{~kg}, m_{2}=60 \mathrm{~kg}$, $m_{3}=30 \mathrm{~kg}, \mu_{1}=15 \mathrm{Ns} / \mathrm{m}, \mu_{2}=20 \mathrm{Ns} / \mathrm{m}, \mu_{3}=30 \mathrm{Ns} / \mathrm{m}$.

Using pole placement methods we obtain the values of the gain matrices $K_{1}, K_{2}, K_{3}$. The RPI sets $\Omega_{U B}^{i}$ are obtained as detailed in (6) and their projections on the position subspace are depicted in Figure 1.a. For the first system a nominal trajectory (2) is constructed and one observes in Figure 1.b that any trajectory affected by disturbance (1) will reside in a tube described by a set as in (7).

\section{OPTIMAL TARGET CONFIGURATION FOR A GROUP OF AGENTS}

Once a robustly positive invariant set (7) around each agent (1) was defined, one can introduce a solution for the collision avoidance problem. Then, in order to describe a

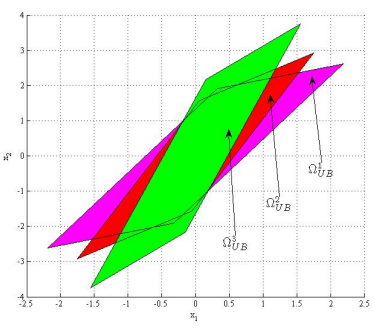

(a)

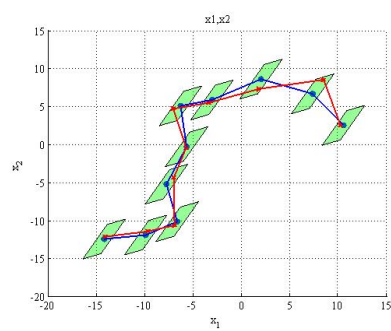

(b)
Fig. 1. (a) Projection of the RPI sets $\Omega_{U B}^{1,2,3}$ on the position subspace, (b) Nominal trajectory and disturbance affected trajectory for the first system.

minimal configuration for the group of agents a mixedinteger optimization problem is solved. The results will be further used such that the agents will converge towards the predefined formation without colliding.

\subsection{Configuration based on representation of RPI sets}

In order to avoid collisions between two different agents $i$ and $j$ inside the formation, it is imposed that

$$
\mathcal{S}_{i}\left(x^{i}\right) \cap \mathcal{S}_{j}\left(x^{j}\right)=\oslash, \quad \forall i, j \in \mathbb{N}_{\left[1, N_{a}\right]}, i \neq j
$$

where the polytope $\mathcal{S}_{i}\left(x^{i}\right) \subset \mathbb{R}^{n}$ (a similar definition can be stated for $\left.\mathcal{S}_{j}\left(x^{j}\right) \subset \mathbb{R}^{n}\right)$ is defined through its implicit half-space description:

$$
\mathcal{S}_{i}\left(x^{i}\right)=\left\{x \in \mathbb{R}^{n}: H_{i}\left(x-x^{i}\right) \leq K_{i}\right\},
$$

with $H_{i} \in \mathbb{R}^{n_{i} \times n}, K_{i} \in \mathbb{R}^{n_{i} \times 1}, n_{i}$ is the number of halfspaces describing the set and $i \in \mathbb{N}_{\left[1, N_{a}\right]}$.

In the sequel, the problem (10) of the intersection for the sets describing safety regions (11) is detailed. To guarantee that two (or more) agents will not superpose one needs to give some conditions on $\left(x^{i}, x^{j}\right)$ for each pair $(i, j) \in \mathbb{N}_{\left[1, N_{a}\right]} \times \mathbb{N}_{\left[1, N_{a}\right]}, i \neq j$, that will make the intersection of their safety regions void. Note that this is important in both static and dynamic settings (as it will be further detailed).

Without entering into an exhaustive presentation, the solution to the problem (10) involves several basic concepts related to parameterized polyhedra ([Loechner and Wilde, 1997], [Olaru and Dumur, 2004]).

The parametrized polytopes are defined in the implicit form by a finite number of inequalities and equalities with the note that the affine part depends linearly on a vector of parameters for both equalities and inequalities. Each of the safety regions (11) are actually parameterized polytopes since are centered "around" their agents, thus depending on a vector of parameters $x^{i} \in \mathbb{R}^{n}, x^{j} \in \mathbb{R}^{n}$ :

$$
\mathcal{S}_{i}\left(x^{i}\right)=\left\{x \in \mathbb{R}^{n}: H_{i} x \leq H_{i} x^{i}+K_{i}\right\},
$$

with $i, j \in \mathbb{N}_{\left[1, N_{a}\right]}$ with $i \neq j$.

As such, the intersection of safety regions $\mathcal{S}_{i}\left(x^{i}\right)$ and $\mathcal{S}_{j}\left(x^{j}\right)$ may be described through a polytope $\mathcal{S}_{i j}(p)$ with $p=\left[\begin{array}{ll}x^{i} & x^{j}\end{array}\right]^{T}$, parameterized by the positions $x^{i}, x^{j}$ of the two agents:

$$
\mathcal{S}_{i j}(p)=\left\{x \in \mathbb{R}^{n}:\left[\begin{array}{l}
H_{i} \\
H_{j}
\end{array}\right] x \leq\left[\begin{array}{l}
K_{i} \\
K_{j}
\end{array}\right]+\left[\begin{array}{cc}
H_{i} & 0 \\
0 & H_{j}
\end{array}\right] p\right\},
$$


with $p \in \mathbb{R}^{2 n}, i, j \in \mathbb{N}_{\left[1, N_{a}\right]}$ and $i \neq j$. In an augmented space, state and parameters space, the parameterized polytope $\mathcal{S}_{i j}(p)$ is identified with a non-parameterized polytope $\tilde{\mathcal{S}}_{i j} \subset \mathbb{R}^{3 n}$ :

$$
\tilde{\mathcal{S}}_{i j}=\left\{\left[\begin{array}{l}
x \\
p
\end{array}\right] \in \mathbb{R}^{3 n}:\left[\begin{array}{ccc}
H_{i} & -H_{i} & 0 \\
H_{j} & 0 & -H_{j}
\end{array}\right]\left[\begin{array}{l}
x \\
p
\end{array}\right] \leq\left[\begin{array}{c}
K_{i} \\
K_{j}
\end{array}\right]\right\}
$$

[Loechner and Wilde, 1997] states that the $p$-dimensional facets of $\tilde{\mathcal{S}}_{i j}$ describe the parameterized vertices of $\mathcal{S}_{i j}$ and their existence domains. It follows that the projection of $\tilde{\mathcal{S}}_{i j}$ on the parameter space describes the region in which at least a parameterized vertex of $\mathcal{S}_{i j}$ exists:

$$
\mathcal{S}_{p}^{i j}=\operatorname{Proj}_{p}\left(\tilde{\mathcal{S}_{i j}}\right) \text {. }
$$

with the complement of the convex region $\mathcal{S}_{p}$ defined as:

$$
\overline{\mathcal{S}_{p}^{i j}}=\operatorname{cl}\left(X \backslash \mathcal{S}_{p}\right)
$$

where $X$ is presumed known or is considered to be the entire space $\mathbb{R}^{n}$.

Finally, it follows that $\overline{\mathcal{S}_{p}^{i j}}$ describes all the pairs $\left(x^{i}, x^{j}\right)$ in the parameter space assuring the fulfillment of (10) for any pair $(i, j) \in \mathbb{N}_{\left[1, N_{a}\right]} \times \mathbb{N}_{\left[1, N_{a}\right]}, i \neq j$.

The above computations can be readily extended to a general case of $N_{a}$ agents. Only the case of a single pair will be further considered, in the interest of compactness.

\subsection{Minimal configuration}

Supposing that the goal is to cluster the agents as closed as possible to the origin, it is desired to obtain a minimal configuration for the group of agents (1), with respect to a given cost function under the constraints described in (10). As a cost function, the sum of the distances of each agent to the origin is chosen, which permits to state the optimization problem:

$$
\min _{x^{i}, i \in \mathbb{N}_{\left[1, N_{a}\right]}} \sum_{i=1}^{N_{a}}\left\|x^{i}\right\|_{2}
$$

subject to: $S_{i} \cap S_{j}=\oslash, \quad \forall i, j \in \mathbb{N}_{\left[1, N_{a}\right]}, i \neq j$.

Following the results in Subsection 3.1 the problem (17) is rewritten as:

$$
\begin{gathered}
\min _{x^{i}, i \in \mathbb{N}_{\left[1, N_{a}\right]}} \sum_{i=1}^{N_{a}}\left\|x^{i}\right\|_{2} \\
\text { subject to: }\left[\begin{array}{c}
x^{i} \\
x^{j}
\end{array}\right] \in \overline{\mathcal{S}_{p}^{i j}}, \quad \forall i, j \in \mathbb{N}_{\left[1, N_{a}\right]}, i \neq j .
\end{gathered}
$$

It can be observed that the constraints introduced in (18) are non-convex. This imposes the use of mixed integer techniques for describing the feasible region, which means that auxiliary binary variables are added to the problem. This is a sensitive numerical problem since the existing branch and cut algorithms employed for solving MIP problems are NP-hard in the number of binary variables. Preserving a linear structure of the constraints, a generic solution for reducing the number of binary variables necessary in describing the exterior of a convex set is proposed in Stoican et al. [2011].

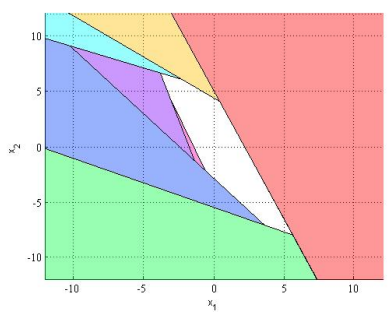

(a)

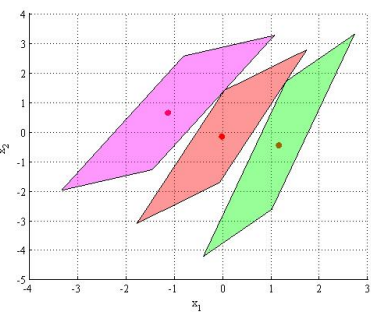

(b)
Fig. 2. (a) The non-convex feasible region (b) The minimal configuration for the three agents

Finally, solving the mixed-integer optimization problem (18), a set of target positions is obtained:

$$
T=\left\{x_{f}^{1}, x_{f}^{2}, \ldots, x_{f}^{N_{a}}\right\}
$$

which describes the minimal configuration under the given cost function.

\subsection{Exemplification for minimal configuration}

Consider the same set of three heterogeneous agents with dynamics described by (8)-(9). Applying the results described in Subsection 3.1 for all possible pairs $(i, j) \in \mathbb{N}_{[1,3]} \times \mathbb{N}_{[1,3]}, i \neq j$ we obtain the feasible regions where no combination of parameters $\left(x^{i}, x^{j}\right)$ will result in an intersection. Figure 2.a depicts a projection on the position subspace of the feasible region. Then, by solving the mixed-integer optimization problem (18) we obtain the set of target positions $T=\left\{x_{f}^{1}, x_{f}^{2}, x_{f}^{3}\right\}$ with $x_{f}^{1}=\left[\begin{array}{lllll}1.15 & -0.46 & 0 & 0\end{array}\right]^{T}, x_{f}^{2}=\left[\begin{array}{llll}-0.02 & -0.16 & 0 & 0\end{array}\right]^{T}$, $x_{f}^{3}=\left[\begin{array}{llll}-1.13 & 0.63 & 0 & 0\end{array}\right]^{T}$, as depicted in Figure 2.b.

\section{OPTIMAL ASSIGNMENT}

The problem described in this section is the optimal assignment problem from the field of combinatorial optimization [Osiadacz et al., 1990], [Hallefjord, 1993]. Giving the results in Subsection 3.2 we want to determine who goes where in the predefined formation. This is equivalent with finding the best permutation over the set of the final positions in the target formation, $T$ from (19).

\subsection{Task assignment formulation}

If a cost $c_{i j}$ is associated to the assignment of agent $j$ to target $x_{f}^{i}$, the problem of finding the best assignment is defined as:

$$
\min _{\delta_{i j}, i, j \in \mathbb{N}_{\left[1, N_{a}\right]}} \sum_{i=1}^{N_{a}} \sum_{j=1}^{N_{a}} c_{i j} \delta_{i j}
$$
subject to:

$$
\sum_{i=1}^{N_{a}} \delta_{i j}=1, \quad \sum_{j=1}^{N_{a}} \delta_{i j}=1, \quad \delta_{i j} \in\{0,1\}, \quad \forall i, j,
$$

where $\delta_{i j}$ are the decision variables ${ }^{1}$ :

$$
\delta_{i j}= \begin{cases}1, & \text { if target } x_{f}^{i} \text { is assigned to agent } j, \\ 0, & \text { otherwise. }\end{cases}
$$

\footnotetext{
1 The constraints in the assignment problem (20) ensure that each agent is assigned to one unique target position.
} 


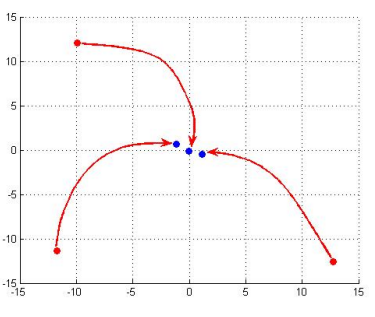

(a)

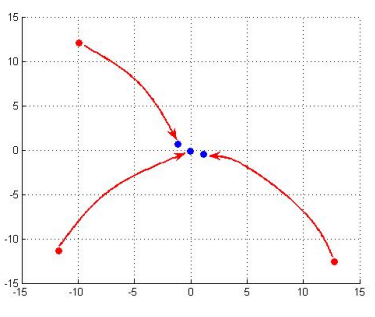

(b)
Fig. 3. (a) Assignment with a non-minimal cost (b) Assignment with a minimal cost

The simplest way is to choose the $\operatorname{cost} c_{i j}$ as the distance between the actual position of agent $j$ and the desired target position in the formation. Hence, the problem would be to determine the minimal distance that an agent has to travel to establish the optimal assignment in the specified formation.

A more insightful way is to use the unconstrained dynamics (2) of the agents to describe the cost of reaching from the initial position to the desired position. Then, $c_{i j}$ can be described by a weighted norm

$$
c_{i j}=\left(x^{j}-x_{f}^{i}\right)^{T} P\left(x^{j}-x_{f}^{i}\right), \quad i, j \in \mathbb{N}_{\left[1, N_{a}\right]}
$$

with the matrix $P=P^{T} \geq 0$ given by the Lyapunov function or the infinite time cost-to-go, as long as the agents follow the unconstrained optimum through the control action:

$$
u^{j}=-K_{j}\left(x^{j}-x_{f}^{i}\right)+\bar{u}^{i}, \forall i, j \in \mathbb{N}_{\left[1, N_{a}\right]}, i \neq j,
$$

where $\bar{u}^{i}$ is chosen such that $x_{f}^{i}=A_{j} x_{f}^{i}+B_{j} \bar{u}^{i}$, with $\bar{u}^{i}=B_{j}^{-1}\left(I-A_{j}\right) x_{f}^{i}$, if the matrix $B_{j}$ is invertible (or the alternative pseudo-inverse which allows the definition of a fixed point for the nominal trajectory).

This optimization problem can be reduced to a simple LP (Linear Programming) problem, hence it can be efficiently computed.

\subsection{Exemplification for optimal assignment}

Consider the set of target positions $T=\left\{x_{f}^{1}, x_{f}^{2}, x_{f}^{3}\right\}$ obtained in Subsection 3.3 and a set of initial positions. Figure 3.a depicts a random assignment and Figure 3.b illustrates an optimal assignment. It can be observed that the cost of reaching the target positions is smaller in the second case.

\section{OPTIMIZATION-BASED CONTROL APPROACH}

After finding the ideal position of the agents in the desired formation the centralized MPC problem is designed based on a two-stage procedure: the optimal assignment which is re-evaluated at each iteration and the mixed-integer optimization problem for driving the group of agents to the specified positions with collision free behavior.

In the following we will refer to the set of $N_{a}$ constrained systems as the global system defined as:

$$
\tilde{x}_{k+1}=A_{g} \tilde{x}_{k}+B_{g} \tilde{u}_{k},
$$

with the corresponding vectors which collects the states and the inputs of each individual nominal system (2) at time $k$, i.e. $\tilde{x}_{k}=\left[x_{k}^{1^{T}}|\cdots| x_{k}^{N_{a}{ }^{T}}\right]^{T}, \tilde{u}=\left[u_{k}^{1^{T}}|\cdots| u_{k}^{N_{a} T^{T}}\right]^{T}$ and the matrices which describe the model: $A_{g}=\operatorname{diag}\left[A_{1}, \cdots, A_{N_{a}}\right], B_{g}=\operatorname{diag}\left[B_{1}, \cdots, B_{N_{a}}\right]$.

So far the subsystems belonging to the global system are completely decoupled. Further on, we consider an optimal control problem for the global system where the cost function and the constraints couple the dynamic behavior of the individual agents. Also, perfect knowledge of each agent dynamics described by equation (2) is available to all the other agents. Consequently, the global model is used in a predictive control context which permits the use of non-convex constraints for collision avoidance behavior.

The receding horizon control is used in order to drive the agents to a target assigned by the optimization problem (20). Also the agents have to avoid collisions along their evolution towards the formation, therefore the constraints will impose that the inherited safety regions associated to each agent do not intersect. In what follows this receding horizon mixed-integer optimization problem is described in detail.

\subsection{Receding horizon optimization problem}

Let $\tilde{x}_{k+l \mid k}$ denote the value of $\tilde{x}$ at time instant $k+l$ predicted at the current time instant $k, k \in \mathbb{N}$. A finite receding horizon implementation is typically based on the solution of an open-loop optimization problem. An optimal control action $\tilde{u}^{*}$ is obtained from the control sequence $\tilde{\mathbf{u}} \triangleq\left\{\tilde{u}_{k \mid k}, \tilde{u}_{k+1 \mid k}, \cdots, \tilde{u}_{k+N-1 \mid k}\right\}$ as a result of the optimization problem:

$$
\begin{gathered}
\tilde{u}^{*}=\arg \min _{\tilde{\mathbf{u}}} V_{N}\left(\tilde{x}_{k \mid k}, \tilde{u}_{k \mid k}\right) \\
\text { subject to: } \\
\left\{\begin{array}{l}
\tilde{x}_{k+l \mid k}=A_{g} \tilde{x}_{k+l-1 \mid k}+B_{g} \tilde{u}_{k+l-1 \mid k}, \quad l \in \mathbb{N}_{[1, N]} \\
x_{k+l \mid k}^{i}, x_{k+l \mid k}^{j} \in \overline{\mathcal{S}_{p}^{i j}}, \forall i, j \in \mathbb{N}_{\left[1, N_{a}\right]}, \quad i \neq j,
\end{array}\right.
\end{gathered}
$$

In order to assure that the target positions (19) are reached the cost function is defined as:

$$
\begin{aligned}
V_{N}\left(\xi_{k \mid k}, \tilde{u}_{k \mid k}\right)= & \xi_{k+N \mid k}^{T} \tilde{P} \xi_{k+N \mid k}+\sum_{l=1}^{N-1} \xi_{k+l \mid k}^{T} \tilde{Q} \xi_{k+l \mid k}+ \\
& +\sum_{l=0}^{N-1} \tilde{u}_{k+l \mid k}^{T} \tilde{R} \tilde{u}_{k+l \mid k}
\end{aligned}
$$

with $\xi_{k} \triangleq \tilde{x}_{k}-x_{f}^{i(k)}$ and $x_{f}^{i(k)}$ represents the optimal target position at current time $k$. Here $\tilde{Q}=\tilde{Q}^{T} \geq 0, \tilde{R}>0$ are the weighting matrices with appropriate dimensions, $\tilde{P}=\tilde{P}^{T} \geq 0$ defines the terminal cost and $N$ denotes the length of the prediction horizon. After introducing $\xi$ in (25), the cost function is reformulated as:

$$
\begin{gathered}
V_{N}\left(\tilde{x}_{k \mid k}, \tilde{u}_{k \mid k}\right)=\tilde{x}_{k+N \mid k}^{T} \tilde{P} \tilde{x}_{k+N \mid k}+\sum_{l=1}^{N-1}\left(\tilde{x}_{k+l \mid k}^{T} \tilde{Q} \tilde{x}_{k+l \mid k}+\right. \\
\left.+2 \tilde{F}^{T} \tilde{x}_{k+l \mid k}\right)+\sum_{l=0}^{N-1} \tilde{u}_{k+l \mid k}^{T} \tilde{R} \tilde{u}_{k+l \mid k},
\end{gathered}
$$

with $\tilde{F}^{T} \triangleq-\left[x_{f}^{i(1)} \cdots x_{f}^{i(k)}\right] \tilde{Q}$. 


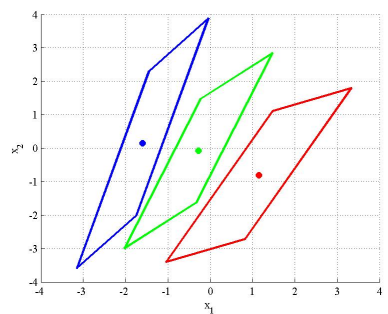

(a)

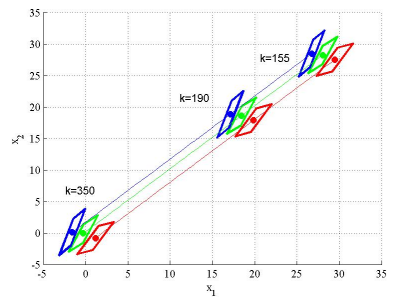

(b)
Fig. 4. (a)Target positions in the minimal configuration (b) Trajectories of the three agents

Through the task assignment mechanism the association between an agent and a target position may change at any moment of time. This means that the matrices defining the cost function must be computed at every moment of time.

\subsection{Exemplification for driving the agents towards the predefined formation}

Consider the three agents described as in (8) with their target positions obtained in Subsection 3.3. We next apply the receding horizon scheme (24) for the global system with a prediction horizon $N=2$. The optimal trajectories for the agents are obtained such that the set of target points is reached through task optimization and under state constraints. The effectiveness of the present algorithm is confirmed by the simulation depicted in Figure 4.b, where the evolution of the agents is represented at three different time instances. The agents successfully reach their target positions in the predefined formation (depicted in Figure 4.a) without violating the constraints.

\section{CONCLUSIONS}

This paper presents first several tools in order to provide a systematic off-line procedure for the control of multiple agents towards a minimal configuration. Second, in realtime a two stage receding horizon control design is adopted for driving the agents to the predefined formation with collision free behavior. At a first stage an optimal assignment problem which is re-evaluated at each iteration is solved in order to find the best association between an agent and the target position within the formation. In a second stage we solve a mixed-integer optimization problem according to the target geometry of the formation and the associated safety regions. Mixed-integer techniques are employed in order to assure a collision free behavior along the evolution of the agents.

\section{REFERENCES}

Beard R.W., McLain T.W., Goodrich M.A. and Anderson E.P. (2002): Coordinated target assignment and intercept for unmanned air vehicles. In Proc. of IEEE Transactions on Robotics and Automation, 18(6):911922 .

Bemporad A. and Morari M. (1999): Control of systems integrating logic, dynamics, and constraints. Automatica 35(3):407-427.
Blake W. and Multhopp D. (1998): Design, performance and modeling considerations for close formation flight. American Institute of Aeronautics and Astronautics Journal.

Blanchini F. and Miani S. (2007): Set-theoretic methods in control. Birkhauser Boston.

Dunbar W.B. and Murray R.M. (2002): Model predictive control of coordinated multi-vehicle formations. In Proc. of the 41th IEEE Conference on Decision and Control, volume 4, pages 4631-4636, Las Vegas, Nevada, USA.

Earl M.G. and D'Andrea R. (2007): A decomposition approach to multi-vehicle cooperative control. Robotics and Autonomous Systems, 55(4):276-291.

Garey M.R. and Johnson D.S. (1979): Computers and intractability. A guide to the theory of NP-completeness. A Series of Books in the Mathematical Sciences. WH Freeman and Company, San Francisco, California.

Hallefjord K.O. (1993): Solving large scale generalized assignment problems. An aggregation/disaggregation approach. European Journal of Operational Research, 64(1):103-114.

Kofman E., Haimovich H. and Seron M.M. (2007): A systematic method to obtain ultimate bounds for perturbed systems. International Journal of Control, 80(2):167178.

Loechner V. and Wilde D.K. (1997): Parameterized polyhedra and their vertices. International Journal of Parallel Programming, 25(6):525-549.

Mayne D.Q., Seron M.M. and Raković S.V. (2005): Robust model predictive control of constrained linear systems with bounded disturbances. Automatica, 41(2):219-224.

Mesbahi M. and Hadaegh F.Y. (2001): Formation flying control of multiple spacecraft via graphs, matrix inequalities, and switching. Journal of Guidance, Control, and Dynamics, 24(2):369-377.

Olaru S. and Dumur D. (2004): A parameterized polyhedra approach for explicit constrained predictive control. In Proc. of the 43th IEEE Conference on Decision and Control, pages 1580-1585, Atlantis, Paradise Island, Bahamas.

Olaru S., De Dona J.A. and Seron M.M. (2008): Positive invariant sets for fault tolerant multisensor control schemes. In Proc. of the 17th IFAC World Congress, pages 1224-1229, Seoul, South Korea.

Osiadacz A.J., Nemhauser G.L. and Wolsey L.A. (1990): Integer and combinatorial optimization. International Journal of Adaptive Control and Signal Processing, 4(4):333-334.

Raković S.V., Kerrigan E.C., Kouramas K.I. and Mayne D.Q. (2005): Invariant approximations of the minimal robust positively invariant set. IEEE Transactions on Automatic Control, 50(3):0 406-410.

Richards A. and How J.P. (2002): Aircraft trajectory planning with collision avoidance using mixed integer linear programming. In Proc. of the 21th American Control Conference, pages 1936-1941, Anchorage, Alaska, USA.

Stoican F., Prodan I. and Olaru S. (2011): On the hyperplanes arrangements in mixed-integer techniques. In Proc. of the American Control Conference. 